\title{
Abortion care training framework for nurses within the context of higher education in the Western Cape
}

I Smit, PhD

Lecturer, Division of Nursing, Faculty of Health Sciences, University of Stellenbosch

\section{EM Bitzer, DEd}

Professor, Department of Curriculum Studies, Faculty of Education, University of Stellenbosch

\section{ELD Boshoff, DEd}

Senior Lecturer, Division of Nursing, Faculty of Health Sciences, University of Stellenbosch

DW Steyn, MD

Professor, Department of Obstetrics and Gynaecology, Faculty of Health Sciences, University of Stellenbosch

\section{Key words}

abortion care training; abortion care training at higher education institutions; certified nurse

\section{Correspondence address \\ Dr llze Smit \\ Division of Nursing \\ Faculty of Health Sciences \\ University of Stellenbosch \\ Tygerberg 7505}

\section{Tel : 0832876372}

Fax : (021) 976-4646

E-mail : ilze.smit(ajxsinet.co.za

\begin{abstract}
Curationis 32 (3): 38-46
The high morbidity and mortality rate due to illegal abortions in South Africa necessitated the implementation of abortion legislation in February 1997. Abortion legislation stipulates that registered nurses who had undergone the proposed abortion care training - certified nurses - may carry out abortions within the first 12 weeks of pregnancy. Currently it seems that an inadequate number of nurses are being trained in the Western Cape to provide pregnant women with counselling, to perform abortions and/or refer problem cases. No real attempts have since been made by higher education institutions in the Western Cape to offer abortion care training for nurses. This case study explores the situation of certified nurses and the context in which they provide abortion care in different regions of the Western Cape. The sampling included a random, stratified (non-proportional) number of designated state health care facilities in the Western Cape, a non-probability purposive sampling of nurses who provided abortion care, a non-probability convenience sample of women who had received abortion care, and a non-probability purposive sampling of final-year pre-registration nursing students. Data was generated by means of questionnaires, a checklist and semi-structured interviews. The main findings of this study indicate that the necessary infrastructure required for legal abortion is in place. However, the ongoing shortage of trained health care practitioners hampers abortion care services. Deficiencies were identified in the existing provincial protocol as some of the guidelines were either not in use or had become obsolete. Certified midwives who had been trained by the regional offices of the Department of Health: Western Cape were skilled in carrying out the abortion procedure, but other aspects of abortion care mainly carried out by other categories of nurses required more attention. This article suggests a training framework that should provide focus for the development of a formal programme or programmes for the training of nurses in abortion care at higher education institutions in the Western Cape.
\end{abstract}




\section{Background}

Act No. 92 of 1996: Choice on Termination of Pregnancy came into effect in South Africa on I February 1997 with the aim of ending illegal abortions and ensuring that unwanted first trimester pregnancies could be legally terminated on request at designated level one health care facilities by certified midwives - those who had completed abortion care training. Despite legislation that permits certified nurses to carry out first trimester abortions, it appears that an inadequate number of nurses who provide counselling to pregnant women, perform abortions and/or have to refer problem cases, are trained in the Western Cape. No formal abortion care training for nurses has been provided by higher education institutions in the Western Cape since abortion legislation came into effect and nurses are trained in abortion care by the Department of Health: Western Cape only.

\section{Problem statement}

There is a serious need for higher education institutions to implement abortion care training. A research gap was identified to develop a comprehensive framework for higher education institutions in the Western Cape to train nurses in abortion care. The prime research question for this study was: What should a higher education training framework for abortion care entail? This led to the following sub-questions:

- What do existing training models or training guidelines entail?

- Who are the role players in abortion care training?

- What is the level of theoretical knowledge and practical skills of nurses to performing abortions on request?

- What are the needs of nurses with regard to training in abortion care?

- What are the needs of women with unwanted pregnancies regarding abortions on request?

- What are the needs of health care facilities with regard to the training of nurses in abortion care?

\section{Methodology}

The study nas mainly of a quantitative nature, but also included qualitative data. A case study design was used as a research strategy and the unit of analysis was the certified nurses within the context in which they provided abortion care. The sampling included:

- $\quad$ a random, stratified sample (non-proportional) of the designated state health care facilities $(N=41 ; n=15)$ representative of all levels of health care facilities in the various regions of the Western Cape;

- a non-probability purposive sample of all the cerrified nurses $(N=15 ; n=10)$ who provided abortion care to ensure that those with specialised knowledge and experience were included;

- a non-probability convenience sample of women who had received abortion care, in order to investigate a particular phenomenon by simply reach [ing] out and tak[ing] the cases that are at hand, continuing the process until the sample reaches a designated size 'or until data saturation w'as reached (De Vos, 2001:198) $(N=12894 ; n=30-100)$; and

- $\quad$ a non-probability purposive sample of pre-registration nursing students $(N=2011$; $n=375$ ). Final-vear nursing students were regarded as being most representative of pre-registration students.

\section{Data collection}

Various data collection methods were used between October 2007 and March 2008 to acquire valid data from different perspectives. Quantitative data was generated by means of questionnaires to women who had had abortions and or counselling, certified nurses who had performed abortions and final-vear pre-registration nursing students. $A$ checklist was used to observe the aborrions that had been performed by certified nurses in an objective and nonparticipatory manner. Qualitative data was obtained by means of semi-structured, individual intervien's with various role plavers in abortion care sen'ices and/or training as w'ell as openended questions in the questionnaire survey (De Vos, 2001:160). The content validity of the checklist and question- naires was increased by basing the items on the set objectives, the guidelines of the World Health Organisation (WHO) and the protocol of the Department of Health: Western Cape. These were supplemented by relevant literature. In order to determine the reliability of the checklist and questionnaires, different observers evaluated the same abortion procedure simultaneously with the same checklist. Guba's model (De Vos, 2001:348) which was used to evaluate the qualitative data refers to four basic criteria, namely truth value, applicability, consistency and neutrality. A pilot study was done prior to the main study, using the various data collection instruments. The necessary amendments were subsequently made. The ethical guidelines that were implemented in this study were based on Uys and Basson's (1991: 106) fundamental guidelines for clinical ethics and the Ethical Standards of Nursing Research (DENOSA, 1998).

\section{Data analysis}

Quantitative data was analysed by means of Statistica 7.0 software, making use of appropriate parametric and/ or non-parametric statistical tests, while qualitative data was analysed using Tesch's analytical approach (De Vos, 2001:343) and Carney's Ladder of Analytical Abstraction (Miles \& Huberman, 1994:92).

\section{Findings}

The main findings of the study as reported below include those which aimed at establishing a higher education framework for abortion care training.

\section{Service provison}

\section{Designated health care facilities}

The necessary infrastructure within which the services could be delivered in compliance with the abortion legislation was adequate at the time of the commencement of this legislation, but registered nurses had not been trained beforehand to provide abortion care services (Dickson-Tetteh \& Billings, 2002:146). The study found that $24 \%$ $(n=10)$ of the designated facilities in the Western Cape did not provide abortion care services, either because of conscientious objection or the non-availability of abortion care practitioners. 
These facilities were obliged to contract services to non-governmental organisations (NGOS), involving additional costs that were not intended when the abortion legislation was implemented. The infrastructure of the facilities that did provide the services were unable to manage the high demand for first trimester abortions and those who were involved in abortion care were inadequately trained.

\section{Integration and decentralisation of services}

Primary health care facilities ought to function as a 'one-stop service' for reproductive health, including abortion care. Despite the additionally designated facilities according to the amended legislation, it seemed that abortion care services had not yet been decentralised or integrated to the entire level one health care, due to the shortage of and in some regions the total absence of nurses who were trained in abortion care.

\section{Referrals}

Despite legislation that stipulates first trimester abortions should be performed by certified nurses at level one health care facilities and referred to appropriate designated level two or three facilities if specialised medical care is deemed necessary, abortions are being performed at all levels of health care which is not cost effective. The study found that approximately $62 \%$ of first trimester abortions are referred to level two facilities from clinics which implies that all nurses should have training in abortion care.

\section{Certified nurses}

\section{General}

In order to deal with the shortage of certified midwives the Amendment Acts No. 38 of 2004: Choice on Termination of Pregnancy and No. 1 of 2008 determine that registered nurses those without qualifications in midwifery and/or community health and/ or primary health care-may also perform abortions if they have completed the proposed course. No registered nurses who had been authorised to undergo abortion care training and perform abortions in compliance with the amended legislation, however, carried out abortion procedures in any of the designated state health care facilities in the Western Cape. The amended legislation had thus made no contribution with regard to the ongoing shortage of certified nurses in the Western Cape. It was found that professional midwives with appropriate and effective training are the ideal category of health practitioner for the provision of abortion care. At the time of the study mainly registered midwives older than 40 years had completed the proposed post-registration course in abortion care with the Department of Health: Western Cape and provided abortion care services at designated state health care facilities. None of these midwives had an advanced qualification in midwifery.

\section{Shortage of certified nurses}

Only 10 of the 15 certified nurses who were in the service of state health care facilities in the Western Cape performed abortions at the time of data collection. Considering that there are 41 designated facilities in the Western Cape that should provide abortion care services according to legislation, this implies that there is a need for more certified nurses who can provide counselling to pregnant women, perform the abortions and refer problem cases.

\section{Workload}

At the time of the study, two certified midwives managed abortions by means of a travelling team on a rotation basis in five and seven designated facilities in the metropolitan area and Boland/Overberg region respectively, while the other certified mid wives carried out other nursing tasks in addition to the abortion care services in their particular facilities. According to the Amendment Act (RSA, 2004:4) the number of designated health care facilities at which abortions must be performed had been increased, but no extra health care practitioners had been provided to manage the additional workload. When any of these nurses were on annual or maternity leave, or resigned, first trimester abortion care services were simply suspended, or delegated to the doctors who were involved with second semester abortions, or they were contracted out to Marie Stopes International - with additional costs. This implies there is indeed a need for more certified nurses.

\section{Needs}

It was found that the certified midwives received no professional, academic or financial recognition for their services and that they could rely on little or no emotional support from the authorities and/or doctors.

\section{Skills}

The relevant certified midwives $(n=10)$ were all trained by the same trainers at the same body, namely the Department of Health: Western Cape. The training. and quality of abortion care provided by them was evaluated according to the guidelines of the World Health Organisation (WHO) (2003), as well as the guidelines of the Department of Health: Western Cape (circular H97/2000). These skills, which relate to pre-abortion care, abortion procedures and postabortion care, are discussed below:

\section{Pre-abortion care}

The study found that the counselling and information sessions were not necessarily presented by certified midwives. They were either presented by registered midwives (44\%) who had not completed the proposed abortion care training, but had qualifications in midwifery and/or community health and/ or primary health care, or by social workers $(36 \%)$. The sessions conducted were mostly presented in groups and they were mainly information sessions that focused on abortion care and not necessarily on resolution counselling. These information sessions were comprehensive, but practically no information was given regarding the manual vacuum aspiration (MVA) procedure.

The pre-abortion (general and obstetrical) assessment of the women was mainly done by registered nurses and midwives who had no abortion care training. It appeared as if these nurses were relied on heavily to provide preabortion care. It further seemed the primary pre-abortion care of women with unwanted pregnancies who requested abortions was not quite satisfactory and that abortions were mostly performed without the women being properly assessed beforehand. The pre-registration training of these registered nurses appeared to be inadequate for this service. The protocol for primary health care regarding the treatment of sexually transmitted diseases and sifting for cervical cancer were, however, well implemented. 


\begin{tabular}{|c|c|c|}
\hline Abortion care & Indicated in protocol & Executed in practice \\
\hline $\begin{array}{l}\text { Pre-abortion care } \\
\text { Resolution counselling } \\
\text { Information and advice } \\
\text { Assessment of patient (history, physical, abdominal and vaginal exami- } \\
\text { nations) } \\
\text { Haematocrit/ haemoglobin } \\
\text { Blood grouping and Rh } \\
\text { Bimanual examination } \\
\text { Sonar } \\
\text { Test for sexually transmitted diseases / sifting cytology } \\
\text { Pre-abortion contraception, emergency contraception }\end{array}$ & $\begin{array}{l}\text { Yes (non-compulsory) } \\
\text { Yes (non-compulsory) } \\
\text { Yes } \\
\text { Yes } \\
\text { Yes } \\
\text { Yes } \\
\text { No } \\
\text { Yes } \\
\text { Yes }\end{array}$ & $\begin{array}{l}\text { Inadequate } \\
\text { Adequate } \\
\text { Inadequate } \\
\\
\text { Inadequate } \\
\text { No } \\
\text { No } \\
\text { Yes } \\
\text { Adequate } \\
\text { Inadequate }\end{array}$ \\
\hline $\begin{array}{l}\text { Abortion procedure } \\
\text { Cervical preparation } \\
\text { MVA procedure } \\
\text { Intra-operative pain relief } \\
\text { Infection control measures } \\
\text { Prophylactic antibiotics }\end{array}$ & $\begin{array}{l}\text { Yes } \\
\text { Yes } \\
\text { Yes } \\
\text { Yes } \\
\text { Yes }\end{array}$ & $\begin{array}{l}\text { Inadequate } \\
\text { Adequate } \\
\text { Inadequate } \\
\text { Inadequate } \\
\text { Inadequate }\end{array}$ \\
\hline $\begin{array}{l}\text { Post-abortion care } \\
\text { Observations } \\
\text { Postoperative pain relief } \\
\text { Postoperative contraception and dual contraception }\end{array}$ & $\begin{array}{l}\text { Yes } \\
\text { Yes } \\
\text { Yes }\end{array}$ & $\begin{array}{l}\text { Inadequate } \\
\text { Inadequate } \\
\text { Adequate }\end{array}$ \\
\hline
\end{tabular}

\section{Abortion procedures}

Ninety-eight $(N=98)$ abortion procedures by health care practitioners were observed in 15 state health care facilities. Only the MVA procedures $(n=73)$ that were carried out by the certified midwives $(n=10)$ were statistically analysed. It appeared that only three of the facilities $(n=3)$ that were visited administered the misoprostol (Cytotec $(B)$ for cervical preparation in compliance with the guidelines of the Department. The administration and dosage of misoprostol differed from region to region and facility to facility. The dosage was also adapted according to the gestation period. The certified midwives were not necessarily involved in the pre-assessment of the women and because of their heavy workload they mostly carried out only' the MVA procedures in the different facilities. It was evident that they were skilled in carrying out the MVA procedure safely and cost effectively at state health care facilities. Despite the guidelines of the Department of Health: Western Cape (2000:8) regarding pain control, it appeared that the medication that was administered was related to either the specific preference of the particular health care practitioner and or the kind of medication that was available in the various facilities. Only 45\% $(n=33)$ of the women who received first trimester abortions received a

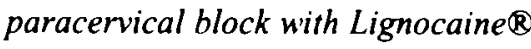
$1 \%$ in compliance with the guidelines for intraoperative pain control, while $50 \%(n=37)$ of the women at a level two facility in the metropolitan area only received an Indocid $($ suppository two hours preoperatively for pain relief. No additional medication for pain relief was offered to these women postoperatively. All the certified midwives followed the protocol of the various facilities regarding infection control the disinfection of instruments, the safe disposal of contaminated articles and sharp objects, as well as the disposal of blood and products of the pregnancy). The nurses' theatre technique with regard to steriliny and/or the 'surgically clean' technique, as well as their protection against the patients ' bodily fluids was, however, inadequate. Notwithstanding the high incidence of $\mathrm{HIV}$ and other sexually transmitted diseases, only $18 \%$ of the women $(n=13)$ who had their pregnancy terminated. received prophylactic antibiorics in compliance with the protocol of the Department of Health: Western Cape (2000:7). It was found that, despite the abovementioned protocol, there was no uniformity in the various regions of the Western Cape in terms of the type of medication, dosage and the administration of prophylactic antibiotics. It appeared that the certified midwives had sufficient knowledge of the syndrome treatment and the administration of the medication, but that medication was not administered because of the heavy daily workload.

\section{Post-abortion care}

The blood pressure of only 29\% of those who underwent abortions was taken after the procedure, while no obsenvations were done with the rest of the women (71\%). Taking into consideration that the MVA is a surgical procedure, it is clear that the aftercare and observations after the procedure were inadequate. Despite studies that found paracetamol to be ineffective in reducing post-abortion pain and that nonsteroidal anti-inflammatory drugs should rather be used, some of the women either received paracetamol (Panado $\$$ or Painblock $($ ) or a combi- 
nation of Brufen $($ and Panado $\otimes$ in compliance with the guidelines of the Department of Health: Western Cape (2000:8), or they were advised to buy it themselves. It appears that the protocol is outdated. Seventy percent (70\%) of the women who underwent abortions received no post-abortion medication for pain relief The certified midwives provided postoperative contraception to all the postoperative women, but in contrast to the policy of the Department of Health: Western Cape (2000:5), dual contraception was not generally provided and/or sufficiently emphasised.

From the summary in Table 1 it is clear that the certified midwives who had been trained in abortion care by the various regional offices of the Department of Health: Western Cape were skilled in performing the MVA procedure, but that the other aspects of abortion care, which are mostly performed by other categories of nurses, needed more attention

\section{Training}

\section{Conscientious objection}

The perception exists that it will be difficult to implement abortion care training in higher education in view of conscientious objection. It was found that 120 (38\%) of the final-year pre-registration students had no conscientious objection to performing abortions. Of these, 36 students $(12 \%)$ indicated that they would complete a post-registration course in abortion care. This implies that there are indeed nurses who can be 'recruited' for abortion care training.

\section{Role players in abortion care training}

Of all the role players who were identified in abortion care training only the International Projects Assistance Services South Africa (IPAS SA), in collaboration with the Department of Health: Western Cape, was directly involved in the formal training of nurses who volunteered for training in abortion care. The South African Nursing Council (SANC) makes no mention of abortion care training for nurses in its regulations or directives. The role of the $S A N C$ with regard to abortion care training was to approve the initial cur- ricula and to certify those who had received the proposed abortion care training. Although short courses are no longer listed by the SANC, the need for short courses is acknowledged.

The SANC has indicated that short courses may be offered by training institutions if such a need has been identified at national or regional level (SANC, 2007). Telephonic surveys (2007) involving the various higher education institutions in the Western Cape indicated that at that time no attempt had yet been made by nursing colleges and/or universities in the Western Cape to implement guidelines and/or formal abortion care training for nurses in the Western Cape.

\section{Existing training guidelines and protocol}

The following abortion care guidelines and protocol are available at present:

1. The WHO manual: Safe Abortion: Technical and Policy Guidance for Health Systems, 2003. These guidelines are based on research findings as well as existing protocol that is used worldwide.

2. The Termination of Pregnancy Care Manual (2002), compiled by IPAS SA in collaboration with the Reproductive Health Research Unit (RHRU), is intended for the training of registered nurses in abortion care.

3. The short course of the Limpopo College of Nursing (2003): Termination of Pregnancy and Related Reproductive Health Issues is based on the initial curriculum as approved by the SANC.

4. A formal course in abortion care training is offered by the various regional offices of the Department of Health: Western Cape (however, owing to a shortage of trainers the course cannot be offered as envisaged).

5. A provincial protocol dealing with abortion care, namely the termination of unwanted pregnancies (H97/2000); the referral of women with specified conditions to a secondary or tertiary facility (H135/1997); and the syndrome treatment of sexually transmitted diseases and other visible infections of the genitals (H84/1995, as amended by circular 80/2008) were implemented by the Department of Health: Western Cape. This makes it imperative for health care practitioners to be trained accordingly in order to be able to deliver an efficient service in compliance with South African abortion legislation and policy.

It appears that various training initiatives do indeed exist, but still an inadequate amount of nurses are being trained in abortion care to supply in the needs of communities.

Overall the study revealed a shortage of trainers and lecturers, as well as a lack of interest in abortion care training among nurses. It furthermore showed that both the pre-registration and post-registration nursing programmes were too full to accommodate additional modules.

\section{Implications of the findings for abortion care trianing}

The recommendations, which are based on a comprehensive literature study as well as on the findings and conclusions that ensued from the empirical part of the study, are contained in a training framework for nurses in abortion care within higher education in the Western Cape. The Learner-centered Curriculum Model (Dolence, 2003) and the Six-step Approach to Curriculum Development (Kern, Thomas, Howard \& Bass, 1998) were used as points of reference for the development of the training framework.

The aim of the training framework, as represented graphically in Figure 1 , is to determine the context within which curriculation must take place, as well as to provide a focus or format for those who develop the curriculum. The training framework is discussed below:

\section{The learner population (frame A)}

Since the study found that there were an insufficient number of certified nurses to perform the service in compliance with abortion legislation, the central focus in the proposed training framework is the learner and her/his learning needs in order to train sufficient nurses effectively in abortion care. The interdependence and reciprocal functioning of the particular frames as proposed in Figure l are regu- 
lated from here.

\section{The learning environment (frame B)}

A positive learning environment, as well as a learner-centered approach aimed at outcomes-based teaching and adult and active learning, has already been established at the various higher education institutions in the Western Cape where the provision of abortion care training is envisaged. No additional provision with regard to lecturers, $i$ brary services, Internet facilities, lecture rooms, study support, advisory services, financial help, accommodation, and health services need necessarily be made if the training is integrated into existing pre-registration and post-registration training programmes. In view of the absence of lecturers in abortion care it would, however, be necessary for the relevant lecturers to undergo additional training, and/or abortion care trainers and/or abortion care providers in the service of the Department of Health: Western Cape and/or IPAS to be contracted to give selected lectures. This inevitably implies extra costs for the institution concerned.

\section{Curriculum design (frame}

\section{C)}

Curriculum design is the first phase of curriculum development when either $a$ new curriculum is being planned or an existing curriculum is re-planned. Decision making features strongly in this phase and is based on a situation analysis and needs assessment in order to provide data for the development of learning outcomes and other activities.

\section{Situation analysis}

Particular attention was given to do a comprehensive situation analysis and needs assessment that could serve as the point of departure to determine: (1) the scope and nature of training and training needs for abortion care at higher education institutions, and (2) the context within which curriculation for abortion care training ought to take place. The different levels at which abortion care training could be offered were investigated, and the needs of the women with unwanted pregnancies, the nurses and the health care facilities re- spectively were determined. As no similar training has been implemented at higher education institutions in the Western Cape since the abortion legislation was passed in February 1997, it is recommended that abortion care training for nurses be implemented as described below:

\section{Integration into existing pre- registration programmes}

Basic abortion care ought to be incorporated in the midwifery and/or community health care component of the pre-registration programme, namely a diploma (National Qualifications Framework $N Q F$ level 6) or the Baccalaureus degree (NQF level 7), by expanding pre-registration modules. The rationale for this recommendation is that these qualyfications would enable a nurse to provide comprehensive health care in compliance with appropriate legislation (SAQA, 2007:3).

\section{Integration into existing post- registration programmes}

A midwife with post-registration qualifications ought to be able to manage most clinical situations due to her/his advanced training. Abortion care should therefore be included as a compulsory module in appropriate postregistration programmes, with the option of completing the practical component. The inclusion of abortion care in specialist courses implies that training be incorporated into either the Advanced Diploma (NQF level 7)-Midwifery and Neonatology or Primary Health Care - or the Baccalaureus Honours degree (NQF level 8). The rationale for this recommendation is to attain specialist knowledge and skills for the provision of reproductive health care (SAQA, 2007:27).

\section{Short course in abortion care}

The existing short course makes provision for abortion care training in view of attaining an Adianced Certificate (NQF level 6) for those who only wish to complete a short course in abortion care, as well as for registered nursesthose without midwifery or related training - who, in compliance with the Amendment Act (RSA, 2004:6), have been authorised to undergo abortion care training. The course, which has been designed as a full-time course, runs over 504 hours and carries 50 cred- its (IPAS SA, 2002:5). The Department of Health: Western Cape and/or higher education institutions that are involved in the post-registration training of nurses should be responsible for offering this short course.

The identification and selection of specific learner outcomes, learning content, teaching and learning activities, as well as assessment and evaluation, take place in accordance with the overarching aim of the curriculum, namely to train nurses in abortion care. These components must be determined within the context and needs of the particular training institution that implements abortion care training.

\section{Learner outcomes}

The curriculum that is developed must reflect a balance between the specific, measurable learner outcomes with associated assessment criteria and critical cross-field outcomes. The former learner outcomes refer to abortion care specific knowledge and the application thereof in the clinical environment, while critical cross-field outcomes refer to general skills such as problemsolving and communication skills, as well as the ability to develop towards lifelong leaming.

\section{Selection of learning content}

Those who develop the curriculum should base the learning content on the selected learner outcomes, supported by the available guidelines as mentioned; appropriate legislation and the protocol of the Department of Health: Western Cape on the termination of pregnancy, but also take cognisance of the findings (empirical and literature review' of this study. Relevant recommendations are made in the following programmes:

\section{Additional learning content in the existing abortion care training}

Based on the findings of the study midwives should be trained in ultrasonography in view of confirming a pregnancy, determining the gestation period and diagnosing an ectopic preg. nancy.

The insertion of the intra-uterine device (IUD) and training in the electric vacuum aspiration ( $E V A$ ) technique for the termination of unwanted pregnancies should be considered. Aspects 


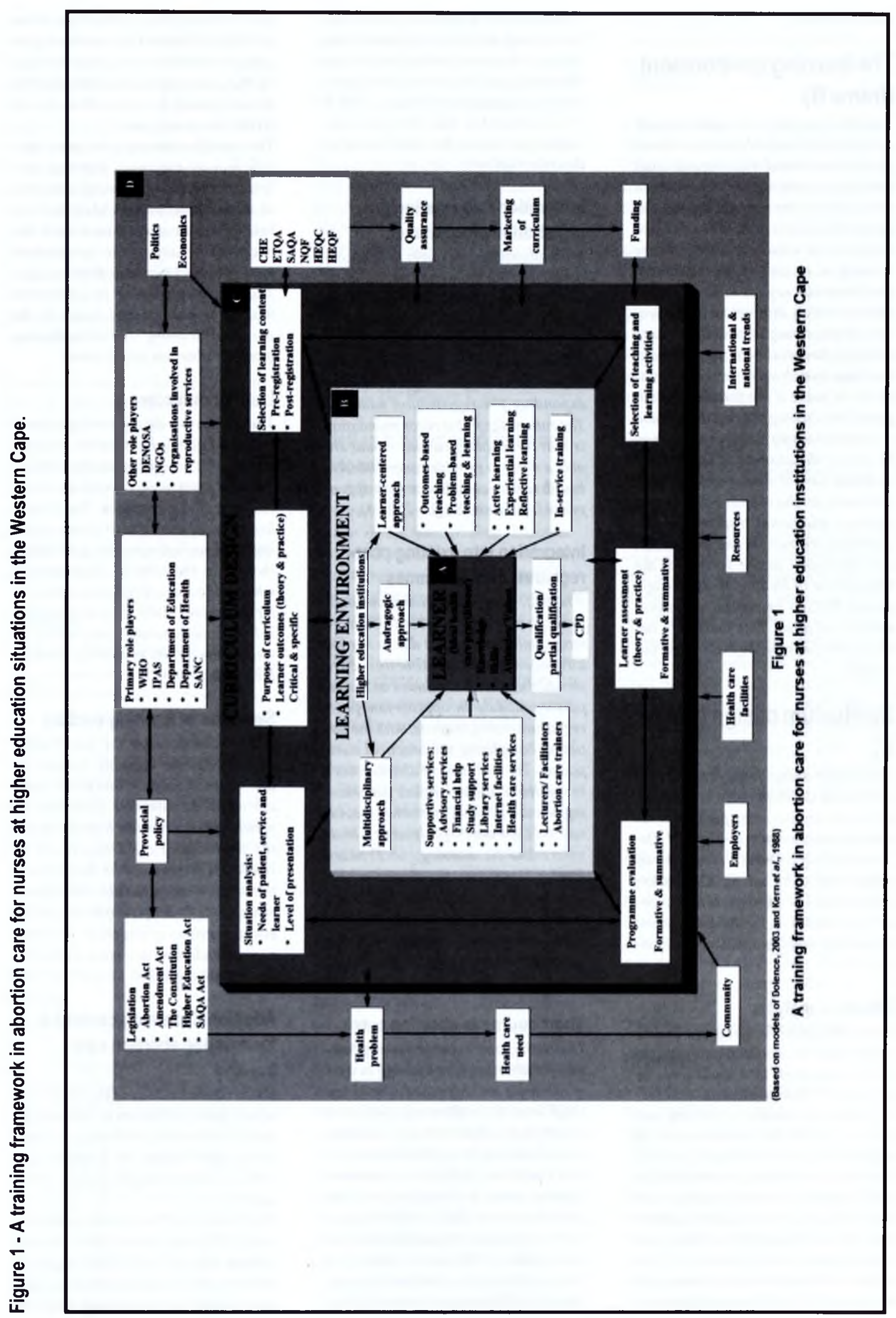


regarding pain relief, infection control, the provision of prophylactic antibiotics for sexually transmitted diseases and record keeping need to be given attention in the existing abortion care training

\section{Recommended abortion care learning content for existing pre-registration programmes}

The amended legislation (RSA, 2004:6) determines that only a registered nurse or midwife may terminate a pregnancy if she/he has completed the proposed training, which implies that pre-registration nursing students may not carry out the practical component of a MVA procedure. The purpose of including abortion care in pre-registration training is, however, to ensure that nurses be exposed to abortion care. In view of the nature of the current pre-registration training and the basic skills that are mastered, abortion care in terms of counselling and information sessions, the pre-abortion assessment of women, post-abortion counselling and contraception could easily be included in preregistration programmes without necessarily having to implement additional modules.

The insufficient skills of registered nurses who have not undergone the proposed abortion care training, but who are involved in the provision of certain aspects of abortion care, need to be addressed by the health care facility involved. These skills mainly entail the history taking and physical examinations, as well as providing contraception and counselling, postoperative observations, postoperative pain control and the emotional care of the women.

\section{Recommended abortion care learning content for existing post-registration programmes}

All aspects of abortion care, including the MVA procedure, should be included in appropriate post-registration programmes with the option that the practical component of this procedure be voluntary for those who have conscientious objections regarding abortions. However, MVA procedures may only be carried out if both the theoretical and the practical components have been completed and assessed.

Owing to the shortage of trainers and absence of lecturers who are trained and/or willing to provide training in addition to their workload, as well as the fact that abortion care is not an isolated field of study, a multidisciplinary approach is indicated involving various role players in the abortion care training of nurses.

\section{Guidelines for the clinical component}

The clinical or practical component is completed at the relevant designated health care facilities in the Western Cape. A minimum of 80 practical hours under the direct supervision of a certified nurse and/or doctor are regarded to be sufficient for a learner to acquire competency in abortion care procedures (IPAS SA, 2002:15).

\section{Determining credits and the recognition of prior learning (RPL)}

As soon as the specific contents have been determined according to which abortion care training is integrated in pre-registration and/or post-registration programmes, credits of the various modules according to notional hours are calculated. In order to prevent the repetition of teaching and learning it is necessary for the relevant training institution to formulate an $R P L$ policy (IPAS SA, 2002:12).

\section{Selection of teaching and learning activities}

The specific teaching and learning activities that are selected are related to the training model that is implemented. The proposed training model is a modular system in which theory and practice are integrated. The theory is centralised at the relevant higher education institution, while practical training is decentralised to designated health care facilities in the Western Cape. Learner-centred teaching and learning methodologies, which are directed towards outcomes-based teaching and based on the concept of adult learning, are recommended. The proposed multidisciplinary approach for abortion care training implies that a wide spectrum of teaching and learning activities will be employed formal lectures, demonstrations, group discussions, case studies, assignments, role play and simulation). Experiential learning opportunities that emphasise real-lyfe scenarios should be included.

\section{The process of assessment and evaluation}

The individual learner's progress ought to be assessed both theoretically and practically by means of formative (continuous) assessment through workplace-integrated evaluation, tests, assignments and/or case studies, while a learner's performance is measured through summative (final) assessment by way of predetermined assessment criteria. The final theoretical assessment should be done by means of $a$ written examination, and formal clinical assessment should take place through objective, structured clinical evaluation (OSCE) by way of set outcomes criteria. Remedial action should be taken when necessary (IPAS SA, 2002:15).

Programme evaluation is necessary to evaluate the programme that has been implemented. This would entail formative evaluation of the programme in order to evaluate the progress of the programme at a given stage. Summative evaluation of the programme should be done of performance measured against the aim that was set for the curriculum.

\section{The infrastructure and role players (frame D)}

The development and implementation of programmes do not take place in isolation. A curriculum is influenced contextually and cannot simply be changed without attention being given to the context within which curriculation takes place. Recommendations for programme development must take place in compliance with the relevant legislation, namely the abortion legislation, the Constitution, the Higher Education Act, the South African Qualifications Authority (SAQA) Act and regulations, the Education White Paper 3, as well as legislation within the National Qualifications Framework (NQF) and the Higher Education Qualifications Framework (HEQF). Various role players, like IPAS SA, the SANC, the Department of Education and quality control bodies such as the Council on Higher Education (CHE), the Education and Training Quality Assurance Body (ETQA), SAQA and the Higher Education Quality Committee (HEQC), also 
have a direct or indirect impact on curriculum development. Other role players that are involved in abortion care, namely health care facilities, NGOs such as Marie Stopes International, DENOSA and organisations involved in reproductive rights such as the Women's Legal Centre, should play a supporting role with regard to the presentation of seminars, workshops and continuing training.

\section{Conclusion}

The study on which this article is based proposes a training framework for the termination of pregnancy for higher education institutions in the Western Cape within which curriculation can take place. The implementation of abortion care training in nursing programmes, whether for diploma of degree purposes, implies that nurses will be empowered academically to deliver comprehensive reproductive services, not only nationally but also internationally, since the proposed abortion care training is based on the guidelines of the WHO (2003) and IPAS $S A$ (2002). The implementation of a formal programme in terms of the proposed training framework should be evaluated, revised and adapted after the completion of the first offering.

\section{References}

DENOSA 1998: Ethical standards for nursing research. Pretoria: DENOSA.

DE VOS, AS 2001: Research at grass roots: A primer for the caring professions. Pretoria: Van Schaik Publishers.

DICKSON-TETTEH, K \& BILLINGS, DL 2002: Abortion care services provided by registered midwives in South Africa. International Familv Planning Perspectives. 28(3):144-150.

DOLENCE, MG 2003: The learnercentered curriculum approach. http:/www.mgdolence.com Accessed 17 June 2008.

IPASSA 2002: Certificate short course: Termination of pregnancy and related reproductive health issues leading to certification as a TOP provider. IPAS.

KERN,DE; THOMAS,PA; HOWARD, DM \& BASS, EB 1998: Curriculum development for medical education.
London: The Johns Hopkins University Press.

MILES, MB \& HUBERMAN, AM 1994: Qualitative data analysis: $A$ sourcebook of new methods. Beverley Hills: Sage.

RSA (REPUBLIC OF SOUTH AFRICA) 1996: Choice on Termination of Pregnancy Act, No. 92 of 1996. Pretoria: Government Printer.

RSA (REPUBLIC OF SOUTH AFRICA) 2004: Choice on Termination of Pregnancy Amendment Act, No. 38 of 2004. Pretoria: Government Printer.

RSA (REPUBLIC OF SOUTH AFRICA) 2008: Choice on Termination of Pregnancy Amendment Act, No. 38 of 2004. Pretoria: Government Printer.

SANC (SOUTH AFRICAN NURSING COUNCIL) 2007: Discontinuation of listing of short courses by the South African Nursing Council. http:// www.sanc.org.za/newsc $702 . \mathrm{htm}$ Accessed 24 April 2007.

SAQA (SOUTH AFRICANQUALIFICATIONS AUTHORITY) 2007: Qualification for public comment. http:// www.saga.org.za Accessed 26 October 2007.

UYS, HHM \& BASSON, AA 1991: Navorsingsmetodologie in die verpleegkunde. Pretoria: HAUM.

\section{WESTERNCAPE DEPARTMENT OF} HEAL TH 2000: Circular No H97/2000: Termination of Pregnancy: Policy, Guidelines and Protocols.

WHO(WORLD HEALTH ORGANISATION) 2003: Safe abortion: Technical and policy guidance for health systems. Geneva: WHO. 